\title{
NOTE ON THE GENERALIZED WHITNEY SUM
}

\author{
ROBERT E. MOSHER ${ }^{1}$
}

1. Introduction. Let $p: E \rightarrow B$ be a Hurewicz fibration with fibre $F$; let $i: F \rightarrow E$ be the inclusion. Then $p$ induces a map $r: E \cup_{i} C F \rightarrow B$ taking $C F$ to the base-point. If we convert $r$ to a fibre map $\pi: E_{r} \rightarrow B$, it is a result of Ganea [2] that the fibre $F_{r}$ has the weak homotopy type of the join $F * \Omega B$, provided $B$ has the homotopy of a CWcomplex.

The purpose of this note is to show that Ganea's fibring may be viewed as the generalized Whitney sum of $E$ with the standard contractible fibring over $B$. In so doing, we generalize a result of Hall [3] and make applicable Hall's theory of Whitney sum fibrings to Ganea's fibration.

2. Statement of the result. Let $P B$ be the space of paths in $B$ ending at the base-point; $P B$ is a contractible fibre space over $B$ with fibre $\Omega B$. Then the generalized Whitney sum [3] of $E$ and $P B$, denoted here $E+P B$, is a fibre space over $B$ with fibre $F * \Omega B$. Let $\rho: E+P B \rightarrow B$ be the projection.

Proposition. Suppose $B$ has the homotopy type of a $\mathrm{CW}$-complex. Then there is a weak homotopy equivalence $v: E+P B \rightarrow E_{r}$ such that $\pi v=\rho$.

Thus, up to weak homotopy type, we may view $E+P B$ as the result of converting $r$ to a fibre map.

Taking $E=P B$, we obtain Hall's result that $P B+P B \rightarrow B$ is, up to weak homotopy type, the Barcus-Meyer fibre sequence $\Omega B * \Omega B$ $\rightarrow S \Omega B \rightarrow B[1]$.

3. Proof. Under the hypotheses, Ganea defines a weak homotopy equivalence $w: F * \Omega B \rightarrow F_{r}$. It suffices by consideration of the homotopy sequences of the fibre maps $\pi$ and $\rho$, to extend $w$ to $v: E+P B \rightarrow E_{r}$ such that $\pi v=\rho$.

Now $E+P B$ is the set of pairs $((1-s) e, s \gamma)$, where $e \in E, 0 \leqq s \leqq 1$, and $\gamma \in P B$ satisfy $\gamma(0)=p(e)$ and are subject to the identifications $(e, 0 \gamma) \sim\left(e, 0 \gamma^{\prime}\right)$ and $(0 e, \gamma) \sim\left(0 e^{\prime}, \gamma\right)$. $\rho$ is defined by $\rho((1-s) e, s \gamma)$ $=p(e)=\gamma(0) . E_{r}$ consists of triples $(e, t, \omega)$ where $0 \leqq t \leqq 1, e \in E$, and $\omega \in B^{I}$ satisfy $p(e)=\omega(1)$, have $t=1$ whenever $e \notin i(F)$, and are subject to the identifications $(e, 0, \omega) \sim\left(e^{\prime}, 0, \omega\right) . \pi$ is defined by $\pi(e, t, \omega)$ $=\omega(0)$.

Received by the editors October 31, 1967.

1 Partially supported by NSF Grant GP-8955. 
Let $\lambda$ be a lifting function [4] for $p: E \rightarrow B$; to a point $e \in E$ and a path $\omega \in B^{I}$ starting at $p(e), \lambda$ associates a path in $E$ covering $\omega$ and starting at $e$. For a path $\omega$, let $\omega_{s}$ be defined by $\omega_{s}(t)=\omega(s t)$. Then $v$ may be defined by

$$
\begin{aligned}
v((1-s) e, s \gamma) & =\left(\lambda\left(e, \gamma_{28}\right)(1), 1, \gamma_{2 s}\right) & & \text { if } 0 \leqq s \leqq \frac{1}{2}, \\
& =(\lambda(e, \gamma)(1), 2-2 s, \gamma) & & \text { if } \frac{1}{2} \leqq s \leqq 1 .
\end{aligned}
$$

That $\pi v=\rho$ is obvious. It remains only to note that the map of $F * \Omega B$ to $F_{r}$ induced by $v$ is precisely the map $w$ given by Ganea.

\section{REFERENCES}

1. W. D. Barcus and J.-P. Meyer, The suspension of a loop space, Amer. J. Math. ${ }^{80}$ (1958), 895-920.

2. T. Ganea, A generalization of the homology and homotopy suspensions, Comment. Math. Helv. 39 (1965), 295-322.

3. I. M. Hall, The generalized Whitney sum, Quart. J. Math. Oxford Ser. (2) 16 (1965), 360-384.

4. W. Hurewicz, On the concept of fiber space, Proc. Nat. Acad. Sci. U.S.A. 41 (1955), 956-961.

California State College at long Beach 